\title{
Reduced-Order Realization of a Nonlinear Power Network Using Companion-Form State Equations with Periodic Coefficients
}

\author{
Taku Noda, Member, IEEE, Adam Semlyen, Life Fellow, IEEE, and Reza Iravani, Senior Member, IEEE
}

\begin{abstract}
This paper presents a methodology for the identification of a reduced-order dynamic equivalent of a nonlinear power network for the simulation of electromagnetic transients. The equivalent is deduced from the observer companion form of the state equations with periodic coefficients and includes the effects of the nonlinearity of the power network at its operating point. A previously proposed concept, the harmonic domain dynamic transfer function (HDDTF), is used to characterize the network's transient behavior, superimposed on the steady state. The HDDTF is obtained by linearization of the nonlinear state equations of the network corresponding to harmonic perturbations applied to the steady-state operating point. Then, reduced-order companion-form state equations with periodic coefficients are fitted to the HDDTF in the frequency domain using a least-squares procedure based on the SVD and QR algorithms. The fitting procedure includes sequential weighting, column scaling, and vertical partitioning to improve computational accuracy and efficiency. The SVD algorithm serves to determine an appropriate model order. A test network with nonlinear inductances is used to demonstrate the performance of the identification method as well as the time-domain simulation results.
\end{abstract}

Index Terms-Dynamics, electromagnetic transient analysis, equivalent circuits, identification, large-scale systems, nonlinear circuits, periodic functions, power system harmonics, saturable cores.

\section{INTRODUCTION}

$\mathbf{T}$ RANSIENT phenomena in ac power networks can overstress their components, and therefore, have to be simulated in order to ensure safe and economic operation [1]-[3]. These networks are however often large and nonlinear and their detailed simulation can be computationally prohibitive. A solution to this problem is to divide the network into two parts: a study zone and an external zone. The study zone is a small portion of the network that includes the source of the transient, and thus, the excursion from the steady state is significant. Therefore, it must be modeled in full detail. The external zone, on the other hand, is the remaining largest part of the network, where an excursion from the steady state is small enough to

Manuscript received April 8, 2002. This work was supported by the Natural Sciences and Engineering Research Council of Canada.

T. Noda is with the Electrical Insulation Department, Central Research Institute of Electric Power Industry, Tokyo 201-8511, Japan (e-mail: takunoda@ieee.org). He is currently a Visiting Scientist at the University of Toronto, Toronto, ON, Canada.

A. Semlyen and R. Iravani are with the Department of Electrical and Computer Engineering, University of Toronto, Toronto, ON M5S 3G4, Canada (e-mail: adam.semlyen@utoronto.ca; iravani@ecf.utoronto.ca).

Digital Object Identifier 10.1109/TPWRD.2003.817802 allow linearization. The linearized network can be replaced with a reduced-order dynamic equivalent to gain computational efficiency with acceptable degree of accuracy of the results. We note that power networks include periodically-switching elements such as power electronics converters, as well as nonlinear elements as the magnetizing circuits of transformers. Because of the nonlinear and the periodically-switching elements, the steady state operation is distorted with harmonics. Therefore, the linearization about the periodic steady state gives linear state equations with periodic coefficients, and the effects of nonlinear and periodically-switching elements at the operating point can be reproduced by replacing the linear state equations with a reduced-order equivalent which preserves the periodic nature of the coefficients.

This paper presents a method for identifying a reduced-order dynamic equivalent of an external zone, which includes nonlinear and periodically-switching elements. The nonlinear state equations of the external zone are first linearized about the periodic steady state. This results in a set of linear state equations with periodic coefficients. Then, a newly developed concept, called harmonic domain dynamic transfer function (HDDTF) [4], is used to characterize the linearized external zone in terms of the frequency response of harmonic perturbations superimposed on the steady state. The HDDTF, representing frequency scan data, is fitted with reduced-order companion-form state equations with periodic coefficients. The fitting procedure is based on the SVD and QR algorithms and includes sequential weighting, column scaling, and vertical partitioning to improve computational accuracy and efficiency. The method employs SVD to determine an appropriate model order without trial and error. This paper also describes a methodology to incorporate the time-periodic equivalent into transient simulations. A test network with nonlinear inductances representing the magnetizing branches of transformers is used to demonstrate the performance of the identification method as well as the time-domain simulation results.

\section{Linear Time-Periodic State Equations AND Their CHARACTERIZATION BY HDDTF}

\section{A. Study Zone and External Zone}

Consider a power network operating in periodic steady state with the base frequency $f_{0}$. The network consists of linear timeinvariant $R, L, C$ components, nonlinear $R, L, C$ components, periodically operating switches, and independent voltage and 
current sources with periodic waveforms. The operating period of the switches and the sources is $T\left(=1 / f_{0}=2 \pi / \omega_{0}\right)$, and therefore, the waveforms of all voltages and currents are $T$-periodic. As shown in Fig. 1, a relatively small portion of the network, in the neighborhood of the source of a transient such as a fault, is called the study zone, and the remaining largest part is called the external zone. Due to the transient, only the study zone is involved in a significant excursion from the steady state, and the excursion in the external zone is small enough to allow linearization about the steady state. The two zones are connected by a port or multiple ports with $N_{p h}$ terminals (phases), and the port voltages to the ground and the port currents injected into the external zone are denoted as $v_{p h}, i_{p h} \in \mathbf{R}^{N_{p h}}$, respectively.

\section{B. Linear Time-Periodic State Equations and HDDTF}

The state equations of the external zone can be derived in the following form [5]:

$$
\begin{aligned}
\frac{d x}{d t} & =f(x, u(t)) \\
y & =g(x, u(t))
\end{aligned}
$$

where $x \in \mathbf{R}^{N^{\prime}}, u, y \in \mathbf{R}^{N_{p h}}$ are state, input, and output vectors, respectively, $f \in \mathbf{R}^{N^{\prime}+N_{p h}} \rightarrow \mathbf{R}^{N^{\prime}}, g \in \mathbf{R}^{N^{\prime}+N_{p h}} \rightarrow$ $\mathbf{R}^{N_{p h}}$ are nonlinear functions, and $N^{\prime}$ the number of state variables in the external zone. Since admittance- and conductancebased formulations are widely used in power system analysis, it is often convenient to consider the port voltages as the input vector and the port currents as the output vector: $u=v_{p h}$ and $y=i_{p h}$. However, any two sets of variables that interface the study and external zones, for instance voltages and powers, can be considered as input and output vectors. In this paper, we assume that the steady-state solution of the network is obtained by (1) and (2) using a harmonic power flow solution; a recent paper [6] reviews many of the best harmonic power flow solution methods presently available.

Assume that a transient originates in the study zone causing excursions of $x, u, y$ from the steady state. The excursions are denoted as $\Delta x, \Delta u, \Delta y$, and linearization of (1) and (2) about the periodic steady-state solution gives

$$
\begin{aligned}
\frac{d(\Delta x)}{d t} & =\frac{\partial f}{\partial x} \Delta x+\frac{\partial f}{\partial u} \Delta u \equiv F_{1 t} \Delta x+F_{2 t} \Delta u \\
\Delta y & =\frac{\partial g}{\partial x} \Delta x+\frac{\partial g}{\partial u} \Delta u \equiv G_{1 t} \Delta x+G_{2 t} \Delta u .
\end{aligned}
$$

Because the coefficient matrices $F_{1 t}, F_{2 t}, G_{1 t}$, and $G_{2 t}$, due to the linearization, are $T$-periodic ${ }^{1}$, the above equations are the $T$-periodic linear state equations representing the external zone. The $T$-periodic variation of the coefficients reflects the effects of nonlinear and periodically-switching elements at their steady state operation.

The previously proposed concept of HDDTF [4] is used to characterize the dynamics of (3) and (4). Based on Floquet theory [7]-[9], it is explained in [4] that the input and output vectors can be expressed as $\Delta u=\Delta u_{t} \mathrm{e}^{s t}$ and $\Delta y=\Delta y_{t} \mathrm{e}^{s t}$ for one particular mode, where $s$ is the complex frequency and

\footnotetext{
${ }^{1}$ In this paper, the subscript " $t$ " is used to indicate that the coefficient is $T$-periodic.
}

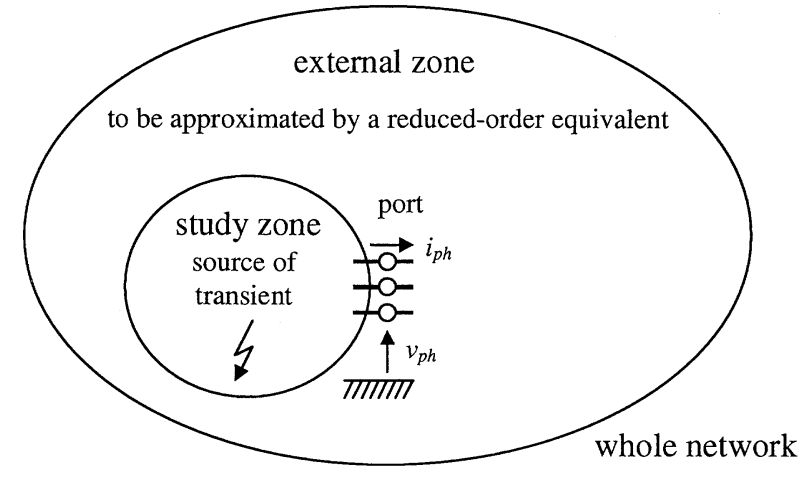

Fig. 1. Study zone and external zone of nonlinear and periodically-switching power network, operating in periodic steady state.

$\Delta u_{t}$ and $\Delta y_{t}$ are their $T$-periodic parts. The $T$-periodic parts are then replaced by their complex Fourier series:

$$
\Delta u_{t}=\sum_{h=-\infty}^{\infty} \Delta u_{h} \mathrm{e}^{j h \omega_{0} t}, \quad \Delta y_{t}=\sum_{h=-\infty}^{\infty} \Delta y_{h} \mathrm{e}^{j h \omega_{0} t} .
$$

Neglecting the $N_{h}$-th and higher order Fourier coefficients, we define the harmonic-domain counterparts of $\Delta u=\Delta u_{t} \mathrm{e}^{s t}$ and $\Delta y=\Delta y_{t} \mathrm{e}^{s t}$ as

$$
\Delta u=\left[\begin{array}{c}
\Delta u_{-\left(N_{h}-1\right)} \\
\vdots \\
\Delta u_{-1} \\
\Delta u_{0} \\
\Delta u_{1} \\
\vdots \\
\Delta u_{N_{h}-1}
\end{array}\right], \quad \Delta y=\left[\begin{array}{c}
\Delta y_{-}\left(N_{h}-1\right) \\
\vdots \\
\Delta y_{-1} \\
\Delta y_{0} \\
\Delta y_{1} \\
\vdots \\
\Delta y_{N_{h}-1}
\end{array}\right] .
$$

Although for simplicity the above harmonic-domain quantities are denoted by the same symbols as the time-domain variables in (3) and (4), clear distinction must be made. The HDDTF matrix $H(s)$, which is derived from (3) and (4) as described in [4], relates the harmonic domain input vector $\Delta u$ to the harmonicdomain output vector $\Delta y$ endowed with $s$-domain properties:

$$
\Delta y=H(s) \Delta u \text {. }
$$

Thus, the HDDTF characterizes the external zone in terms of the frequency response of the harmonic perturbations superimposed on the periodic steady state, in other words, in terms of the transient behavior superimposed on the periodic steady state in the time domain. The HDDTF of the external zone is calculated in the form of frequency-scan data at discrete frequency points $s_{p}=j \omega_{p}(p=1,2, \ldots, P)$ to be used in the subsequent identification process.

\section{REDUCED-ORDER EQUIVALENT}

\section{A. Companion Form Model with Periodic Coefficients}

Our objective is to identify a reduced-order dynamic equivalent from the frequency-scan data of HDDTF. The observer companion form (CF) of state equations with periodic coefficients is used as the equivalent:

$$
\frac{d(\Delta x)}{d t}=A_{t} \Delta x+B_{t} \Delta u
$$


where the state vector $\Delta x$ and the $T$-periodic coefficients $A_{t}$ and $B_{t}$ are given by

$$
\begin{aligned}
& \Delta x=\left[\begin{array}{lllll}
\Delta x_{1} & \Delta x_{2} & \cdots & \Delta x_{N-1} & \Delta x_{N}
\end{array}\right]^{T} \\
& A_{t}=\left[\begin{array}{ccccc}
0 & & & & -a_{0}(t) \\
1 & 0 & & & -a_{1}(t) \\
& 1 & \ddots & & \vdots \\
& & \ddots & 0 & -a_{N-2}(t) \\
& & & 1 & -a_{N-1}(t)
\end{array}\right] \\
& B_{t}=\left[\begin{array}{lllll}
b_{0}(t) & b_{1}(t) & \cdots & b_{N-2}(t) & b_{N-1}(t)
\end{array}\right]^{T} .
\end{aligned}
$$

The functions $a_{0}(t), \ldots, a_{N-1}(t)$ and $b_{0}(t), \ldots, b_{N-1}(t)$ are $T$-periodic. The order of the equivalent $N$ is smaller than the order $N^{\prime}$ of the initial system (3) and (4). The output equations are

$$
\Delta y=C \Delta x, \quad C=\left[\begin{array}{llll}
0 & \cdots & 0 & 1 \cdots 1
\end{array}\right] .
$$

The size of $C$ is $1 \times N N_{p h}$ and the number of 1's at the tail portion is $N_{p h}$. By defining a matrix of dynamic differentiation with respect to time, in the harmonic domain, as

$$
S=\operatorname{diag}\left(\left\{s+j h \omega_{0}\right\} I\right)
$$

where $h=-\left(N_{h}-1\right), \ldots,-1,0,1, \ldots, N_{h}-1$ and $I$ the $N \times N$ identity matrix, the time domain state equations (8) are transformed into the harmonic domain [4]:

$$
S \Delta x=A \Delta x+B \Delta u .
$$

The coefficient matrices $A$ and $B$ in (14) are given by

$$
\begin{aligned}
A & =\left[\begin{array}{ccccc}
0 & & & & -A_{0} \\
I & 0 & & & -A_{1} \\
& I & \ddots & & \vdots \\
& & \ddots & 0 & -A_{N-2} \\
& & & I & -A_{N-1}
\end{array}\right] \\
B & =\left[\begin{array}{lllll}
B_{0} & B_{1} & \cdots & B_{N-2} & B_{N-1}
\end{array}\right]^{T}
\end{aligned}
$$

where $A_{0}, \ldots, A_{N-1}$ and $B_{0}, \ldots, B_{N-1}$ are Hermitian Toeplitz matrices, consisting of the Fourier coefficients of $a_{0}(t), \ldots, a_{N-1}(t)$ and $b_{0}(t), \ldots, b_{N-1}(t)$. For instance, $A_{0}$ takes the following form:

$$
\left[\begin{array}{ccccccc}
a_{0,0} & a_{0,-1} & a_{0,-2} & & & & \\
a_{0,1} & a_{0,0} & a_{0,-1} & \cdot & & & \\
a_{0,2} & a_{0,1} & a_{0,0} & & \cdot & & \\
& \cdot & & \cdot & & \cdot & \\
& & \cdot & & a_{0,0} & a_{0,-1} & a_{0,-2} \\
& & & a_{0,1} & a_{0,0} & a_{0,-1} \\
& & & & a_{0,2} & a_{0,1} & a_{0,0}
\end{array}\right] .
$$

This shows the Hermitian Toeplitz structure of $A_{0}$ with $a_{0, k}$ being the $k$-th Fourier coefficient of $a_{0}(t)$, where $N_{k}=3$ and higher coefficients are truncated. Note that $a_{0, k}=a_{0,-k}^{*}$; the asterisk denotes complex conjugate. The harmonic-domain output equation has the same form as (12) but with vectors of larger size. The purpose of obtaining the harmonic-domain state equations (14)-(16) is to identify the $T$-periodic coefficients $a_{0}(t), \ldots, a_{N-1}(t)$ and $b_{0}(t), \ldots, b_{N-1}(t)$ in terms of their Fourier coefficient matrices $A_{0}, \ldots, A_{N-1}$ and $B_{0}, \ldots, B_{N-1}$.

Equations (3) and (4) can result in the observer CF using an equivalence transformation, provided that the transformation matrix is nonsingular and continuously-differentiable with respect to time [8]. Therefore, the observer CF model with periodic coefficients (8)-(12) is general and can represent any linear, time-periodic system expressed in the form of (3) and (4). This indicates that any linear, time-periodic system can be fitted with the observer CF. Of course, the equivalence transformation of (3) and (4) of the given external zone is not intended to be performed here since it simply gives an observer $\mathrm{CF}$ of unreduced order.

\section{B. Rational-Function Representation of CF Model}

Substituting the state variables $\Delta x$ of (14) into the output equation gives

$$
\begin{aligned}
& \Delta y=\left(A_{0}+S A_{1}+\cdots+S^{N-1} A_{N-1}+S^{N}\right)^{-1} \times \\
& \times\left(B_{0}+S B_{1}+\cdots+S^{N-1} B_{N-1}\right) \Delta u
\end{aligned}
$$

where " $x$ " denotes continuation. Because this is a harmonicdomain input-output relation, the following rational function in $S$ is an HDDTF:

$$
\begin{aligned}
H(S) & \equiv\left(A_{0}+S A_{1}+\cdots+S^{N-1} A_{N-1}+S^{N}\right)^{-1} \times \\
& \times\left(B_{0}+S B_{1}+\cdots+S^{N-1} B_{N-1}\right) \\
& =\left(\sum_{n=0}^{N-1} S^{n} A_{n}+S^{N}\right)^{-1}\left(\sum_{n=0}^{N-1} S^{n} B_{n}\right) .
\end{aligned}
$$

This is the rational-function representation of the observer $\mathrm{CF}$ model (8)-(12) and is employed for identifying the Fourier coefficient matrices $A_{0}, \ldots, A_{N-1}$ and $B_{0}, \ldots, B_{N-1}$ in the next section. The controller variant of $\mathrm{CF}$, that results in another form of the rational function with the inverse of its "denominator" as a right, rather than left, multiplier as in (19), could also be used. However, the use of the controller CF complicates the implementation of sequential weighting described in the next section because of the right multiplication. Therefore, the observer $\mathrm{CF}$ model is used and hereafter simply referred to as "CF model."

\section{IDENTIFICATION OF CF MODEL}

\section{A. Least-Squares Formulation and Sequential Weighting}

We now formulate a least-squares problem to identify the Fourier coefficient matrices $A_{n}$ and $B_{n}(n=0,1, \ldots, N-1)$ of the rational function (19). A first and simple formulation would be to minimize the following objective function, the difference of frequency responses between the rational function model (19) and the given HDDTF matrix $H_{p}$ in (7) at discrete frequency points $\omega_{p}(p=1,2, \ldots, P)$

$$
\sum_{p=1}^{P}\left\|\left(\sum_{n=0}^{N-1} S_{p}^{n} A_{n}+S_{p}^{N}\right)^{-1}\left(\sum_{n=0}^{N-1} S_{p}^{n} B_{n}\right)-H_{p}\right\|_{F}^{2}
$$

where $\|\cdot\|_{F}$ denotes the Frobenius norm and $S_{p}=\left.S\right|_{s=j \omega_{p}}$. Due however to the presence of the "denominator" coefficients $A_{n},(20)$ leads to a nonlinear least-squares problem requiring an 
initial solution. To avoid the nonlinearity, the objective function is linearized by premultiplying by the denominator:

$$
\sum_{p=1}^{P}\left\|\left(\sum_{n=0}^{N-1} S_{p}^{n} B_{n}\right)-\left(\sum_{n=0}^{N-1} S_{p}^{n} A_{n}+S_{p}^{N}\right) H_{p}\right\|_{F}^{2} .
$$

In this objective function, all coefficients to be identified are part of a linear relation, and thus, it results in a linear least-squares problem. However, the objective function is now biased due to the premultiplication by the denominator, in other words, the equations are weighted by the denominator. To cancel this bias, an iterative procedure, which updates a weighting matrix $W$ approximating the inverse of the denominator, is introduced. At the initial iteration step $(m=0)$, the weighting matrix is set to the identity matrix, and at the subsequent $m$-th iteration step $(m=1,2, \ldots)$, it is given by

$$
W_{p}^{(m)}=\left(\sum_{n=0}^{N-1} S_{p}^{n} A_{n}^{(m-1)}+S_{p}^{N}\right)^{-1}
$$

where $A_{n}^{(m-1)}$ are identified coefficient matrices at the preceding iteration step. Thus, the objective function to be minimized is (23) at the bottom of the page, and the identification of $A_{n}^{(m)}$ and $B_{n}^{(m)}$ minimizing (23) is repeated for $m=$ $0,1,2, \ldots$ until the solution converges. This least-squares formulation involves iteration but no initial solution. Compared with (20), the most advantageous fact is that (23) requires no initial solution.

Next, we formulate the overdetermined linear equations to be solved at each iteration step. For each frequency point $\omega_{p}$, (23) gives

$$
\begin{aligned}
\sum_{n=0}^{N-1} W_{p}^{(m)} S_{p}^{n} A_{n}^{(m)} H_{p}-\sum_{n=0}^{N-1} W_{p}^{(m)} S_{p}^{n} B_{n}^{(m)} & \\
& \cong-W_{p}^{(m)} S_{p}^{N} H_{p}
\end{aligned}
$$

where $H_{p}$ is given, $S_{p}$ and $W_{p}^{(m)}$ are known, and $A_{n}^{(m)}$ and $B_{n}^{(m)}$ are unknowns. If it is desirable to apply different weighting for different frequency points, the following expression which introduces the weighting $w_{p}$ is used instead of (24):

$$
\begin{aligned}
\sum_{n=0}^{N-1} w_{p} W_{p}^{(m)} S_{p}^{n} A_{n}^{(m)} H_{p}-\sum_{n=0}^{N-1} w_{p} W_{p}^{(m)} S_{p}^{n} B_{n}^{(m)} \\
\\
\cong-w_{p} W_{p}^{(m)} S_{p}^{N} H_{p} .
\end{aligned}
$$

Since the algebraic relation with respect to $A_{n}^{(m)}$ and $B_{n}^{(m)}$ is linear, (24) or (25) can be brought to the following form using all elements of the matrix equation for all frequency points (see Appendix A for details):

$$
A x \cong b
$$

where matrix $A$ and vector $b$ consist of elements of $H_{p}, S_{p}$, and $W_{p}^{(m)}$ [also $w_{p}$ in the case of (25)], and the unknown vector $x$ consists of distinct elements of $A_{n}^{(m)}$ and $B_{n}^{(m)}$.

\section{B. Column Scaling}

It is well known that a least-squares problem for polynomial or rational fitting often results in an ill-conditioned $A$ matrix in (26). Therefore, prior to the solution of (26), column scaling which balances the Euclidean norms of the columns of $A$ to unity is applied to improve its condition number [10]-[13]. The column scaling is formally expressed by introducing a diagonal matrix $D$ in (26):

$$
A D D^{-1} x \cong b, \quad D=\operatorname{diag}\left(\frac{1}{\left\|a_{j}\right\|_{2}}\right)
$$

where $a_{j}$ is the $j$-th column vector of $A$ and $\|\cdot\|_{2}$ denotes the 2-norm. If we define $A^{\prime} \equiv A D$ and $x^{\prime} \equiv D^{-1} x$, (27) results in $A^{\prime} x^{\prime} \cong b$ which is of the same form as (26). Thus, (27) can be solved in the same way as (26) but with a better condition number. Finally, the solution of (26) is obtained by the solution of $A^{\prime} x^{\prime} \cong b$ using $x=D x^{\prime}$.

\section{Determination of Model Order Using SVD}

At the initial iteration step ( $m=0)$ only, singular value decomposition (SVD) [10], [11] is applied to (26) after column scaling in order to determine an appropriate model order. The $m \times n$ matrix $A$ of (26) is decomposed into an $m \times n$ orthonormal matrix $U$, an $n \times n$ diagonal matrix $\Sigma$, and an $n \times n$ orthonormal matrix $V^{T}$ :

$$
A=U \Sigma V^{T} .
$$

The diagonal matrix $\Sigma$ contains the singular values $\sigma_{1}, \sigma_{2}, \ldots, \sigma_{n}$ of $A$ in nonincreasing order. We define the following transformations of $x$ and $b$ :

$$
y \equiv V^{T} x, \quad g \equiv U^{T} b .
$$

Substituting (28) and (29) into (26) gives

$$
\Sigma y=g
$$

so that

$$
y=\left[\begin{array}{llll}
\frac{g_{1}}{\sigma_{1}} & \frac{g_{2}}{\sigma_{2}} & \cdots & \frac{g_{n}}{\sigma_{n}}
\end{array}\right]^{T} .
$$

Each singular value represents the contribution of the corresponding equation (row) to the solution. To use this information for the model order determination, we first normalize the singular values with respect to $\sigma_{1}: \bar{\sigma}_{1}=1, \bar{\sigma}_{2}=\sigma_{2} / \sigma_{1}, \ldots, \bar{\sigma}_{n}=\sigma_{n} / \sigma_{1}$ and define $\varepsilon$ as a user-defined relative error permitted for the equivalent. Assume that the first $n^{\prime}(<n)$ singular values $\bar{\sigma}_{1}, \ldots, \bar{\sigma}_{n^{\prime}}$ are larger than $\varepsilon$, say $\varepsilon=0.01$ for $1 \%$ error, and the remaining $\bar{\sigma}_{n^{\prime}+1}, \ldots, \bar{\sigma}_{n}$

$$
\sum_{p=1}^{P}\left\|W_{p}^{(m)}\left(\sum_{n=0}^{N-1} S_{p}^{n} B_{n}^{(m)}\right)-W_{p}^{(m)}\left(\sum_{n=0}^{N-1} S_{p}^{n} A_{n}^{(m)}+S_{p}^{N}\right) H_{p}\right\|_{F}^{2}
$$


are smaller. Since the contributions of the smaller singular values are less than $\varepsilon$, the corresponding equations can be eliminated. To this end, the minimum norm solution for $x$ is used in most SVD applications [10], [11]. However, in this particular application, in order to obtain a sparse vector $x$, we use the following solution:

$$
\hat{x}=\left(\hat{V}^{T}\right)^{-1} \hat{y}
$$

where $\hat{V}$ is the left upper $n^{\prime} \times n^{\prime}$ square portion of $V$ and

$$
\hat{y}=\left[\begin{array}{llll}
\frac{g_{1}}{\sigma_{1}} & \frac{g_{2}}{\sigma_{2}} & \cdots & \frac{g_{n^{\prime}}}{\sigma_{n^{\prime}}}
\end{array}\right]^{T} .
$$

Note that the number of elements in the unknown vector $x$ in the initial equations (26) is $n$, while the above solution $\hat{x}$ in (32) gives $n^{\prime}$ elements. This implies that the $\left(n^{\prime}+1\right)$-th to $n$-th elements of $x$ are now forced to be zero and $\hat{x}$ gives a lower order model if the model coefficients are ordered in an appropriate sequence in $x$. As described in Appendix A, the model coefficients $A_{n}$ and $B_{n}$ are in descending order in the initial unknown vector $x$. Thus, for instance, if the lowest three sets of coefficients $A_{0}$, $B_{0}, A_{1}, B_{1}, A_{2}, B_{2}$ located at the tail portion of $x$ are forced to be zero, the factor $S_{p}^{3}$ cancels out at both sides of (24) to result in a lower order model. It should also be noted that each Fourier coefficient matrix $A_{n}$ or $B_{n}$ consists of $2 N_{k}-1$ distinct Fourier coefficients of $a_{n}(t)$ or $b_{n}(t)$ as shown in (17), and the number of Fourier coefficients required to represent one order (a set of a denominator and a numerator coefficient) is $4 N_{k}-2$. Because of this, $n^{\prime}$ must be a multiple of $4 N_{k}-2$ and is practically adjusted to the closest multiple of $4 N_{k}-2$. If the network exhibits half-wave symmetry, the odd-harmonic entries in $A_{n}$ and $B_{n}$ can be set to zero, and thus, $n^{\prime}$ must be a multiple of $2\left(N_{k}-1\right)$ instead.

When the overdetermined linear equations (26) are built at the initial iteration step $(m=0)$, we set the initial model order $N$ to an acceptable maximum order for a satisfactory equivalent. Then the above SVD procedure determines an appropriate lower order, given by $N=n^{\prime} /\left(4 N_{k}-2\right)$, according to a user-defined relative error $\varepsilon$. The solution $\hat{x}$ in (32) is used as the initial solution in the subsequent iterative solution procedure using the $\mathrm{QR}$ decomposition algorithm.

\section{Iterative Solution Procedure Using $Q R$}

The order $n^{\prime} /\left(4 N_{k}-2\right)$ and the initial solution $\hat{x}$ determined in the above SVD procedure are now denoted by $N$ and $x^{(0)}$, respectively, and we enter the subsequent iteration steps $(m=1,2, \ldots)$. The weighting matrix $W_{p}^{(m)}$ in (22) is updated using the solution $x^{(m-1)}$ at the preceding iteration step, and the overdetermined linear equations (26) are rebuilt with the updated weighting matrix. After column scaling, the QR decomposition [10], [11] is applied to (26):

$$
Q R x \cong b \text {. }
$$

Then, $Q^{T}$ is premultiplied to both sides:

$$
R x=Q^{T} b .
$$

The solution is obtained by backward substitution in (35). Because the order has been minimized at the initial SVD step to eliminate redundant unknowns, the newly built $A$ matrix in (26) does not have small singular values which would not contribute to the solution and the elimination of the small singular values is unnecessary. Therefore, the $\mathrm{QR}$ decomposition algorithm is used here due to its superior computational efficiency compared to SVD.

The above QR-based solution procedure is iterated until it converges. Based on (23), the residual vector $r$ is defined as follows. The residual vector $r^{(m)}$ at the $m$-th iteration step is a real-valued column vector with $2 P\left(2 N_{h}-1\right)^{2}$ elements such that all elements of the following matrix $R_{p}^{(m)}$ for all frequency points $(p=1,2, \ldots, P)$ are rearranged into the elements of $r^{(m)}$, where the real and imaginary parts of each element of $R_{p}^{(m)}$ are considered as two independent entries of $r^{(m)}$ :

$$
\begin{aligned}
R_{p}^{(m)}=W_{p}^{(m)}\left(\sum_{n=0}^{N-1} S_{p}^{n} B_{n}^{(m)}\right) \\
\quad-W_{p}^{(m)}\left(\sum_{n=0}^{N-1} S_{p}^{n} A_{n}^{(m)}+S_{p}^{N}\right) H_{p} .
\end{aligned}
$$

By defining the residual $\rho$ as the 2-norm of $r, \rho=\|r\|_{2}$, the convergence can reasonably be detected by the relative difference of $\rho$ between the preceding and present steps (i.e., when $\left|\left(\rho^{(m)}-\rho^{(m-1)}\right) / \rho^{(m)}\right|$ becomes smaller than a user defined constant, the iteration is terminated and the solution at the terminated step is regarded as the final solution).

\section{E. Best Step}

To improve the convergence of the QR-based iterative solution procedure, a scheme to estimate the best step for updating the solution is introduced. The solution vector $x$ of (35) is linearly interpolated with respect to a parameter $\theta$ using the preceding $(m-1)$ and present $(m)$ iteration steps:

$$
x=(1-\theta) x^{(m-1)}+\theta x^{(m)}
$$

where $\theta=0$ gives the preceding solution $x^{(m-1)}$ and $\theta=1$ the present solution $x^{(m)}$. We assume that the residual vector $r$ can also be approximated by a linear interpolation with the same parameter:

$$
r=(1-\theta) r^{(m-1)}+\theta r^{(m)} .
$$

The value of $\theta$ that minimizes the square of $\rho$ is obtained by setting $\partial \rho^{2} / \partial \theta=0$ :

$$
\theta_{\text {best }}=\frac{r^{(m-1)^{T}} r^{(m-1)}-r^{(m-1)^{T}} r^{(m)}}{r^{(m-1)^{T}} r^{(m-1)}-2 r^{(m-1)^{T}} r^{(m)}+r^{(m)^{T}} r^{(m)}} .
$$

After calculating the solution vector $x^{(m)}$ by solving (26) with (22), the value of $\theta_{\text {best }}$ is calculated by (39) and substituted into (37) to obtain an improved solution $x_{\text {best }}$. Finally, the improved solution $x_{\text {best }}$ replaces the present solution $x^{(m)}$. In a simplified two-dimensional (2-D) case where $x$ has only two elements, contours of $\rho^{2}$ in the solution plane are shown in Fig. 2. $\theta_{\text {best }}$ gives the solution point on the straight line of $\theta$ where the gradient of $\rho^{2}$ is perpendicular to the line. Thus, the best step avoids unnecessary overshooting during the iterations.

\section{F. Vertical Partitioning}

If the number of equations in (26) is too large to fit in computer memory, vertical partitioning is applied to reduce 


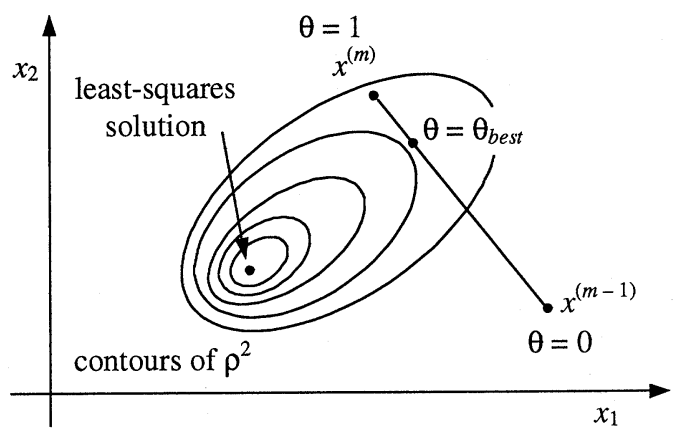

Fig. 2. Contours of $\rho^{2}$ in the solution plane in a simplified 2-D case.

the number of equations. Also for vertical partitioning, the initial iteration step ( $m=0$ ) employs SVD to eliminate less significant equations and the subsequent steps $(m=1,2, \ldots)$ use QR.

After column scaling, (26) is divided into $K$ partitions

$$
\left[\begin{array}{c}
\frac{A_{1}}{A_{2}} \\
\frac{\vdots}{A_{K}}
\end{array}\right] x \cong\left[\begin{array}{c}
\frac{b_{1}}{b_{2}} \\
\vdots \\
\frac{b_{K}}{}
\end{array}\right] .
$$

The numbers of rows in the partitions can be nonuniform, and $A_{k}$ and $b_{k}$ of the $k$-th partition $A_{k} x \cong b_{k}$ are an $m_{k} \times n_{k}$ matrix and a column vector with $m_{k}$ elements, respectively.

At the initial iteration step, SVD is applied to each partition:

$$
U_{k} \Sigma_{k} V_{k}^{T} x \cong b_{k} \text {. }
$$

Assume, in the same way as in Section IV-C, that the first $n_{k}^{\prime}$ $\left(<n_{k}\right)$ normalized singular values are larger than $\varepsilon$ and the remaining singular values are smaller. Then, in (41), only the first $n_{k}^{\prime}$ equations (rows) are meaningful and the equations corresponding to the smaller singular values are ignored. Thus, we define $\hat{\Sigma}_{k}$ as the $n_{k}^{\prime} \times n_{k}^{\prime}$ left upper part of $\Sigma_{k}, \hat{V}_{k}$ and $\hat{U}_{k}$ as the first $n_{k}^{\prime}$ columns of $V_{k}$ and $U_{k}$, respectively. The $U_{k}$, $\Sigma_{k}, V_{k}$ matrices in (41) are replaced by the shrunk counterparts $\hat{U}_{k} \hat{\Sigma}_{k} \hat{V}_{k}^{T} x \cong b_{k}$, and $\hat{U}_{k}^{T}$ is premultiplied to both sides:

$$
\hat{\Sigma}_{k} \hat{V}_{k}^{T} x=\hat{U}_{k}^{T} b_{k} .
$$

Finally, we define $\hat{A}_{k} \equiv \hat{\Sigma}_{k} \hat{V}_{k}^{T}$ and $\hat{b}_{k} \equiv \hat{U}_{k}^{T} b_{k}$, and all shrunk equations $\hat{A}_{k} x=\hat{b}_{k}(k=1,2, \ldots, K)$ are reassembled into the form (40). The number of equations in the shrunk form is reduced to just $n_{1}^{\prime}+\cdots+n_{K}^{\prime}$, which is usually significantly smaller than the number of equations in the initial (40).

At the subsequent iteration steps, QR decomposition is applied to each partition:

$$
Q_{k} R_{k} x \cong b_{k}
$$

Then, $Q_{k}^{T}$ is premultiplied to both sides:

$$
R_{k} x=Q_{k}^{T} b_{k} .
$$

In the same way as in the SVD case, we define $\hat{A}_{k} \equiv R_{k}$ and $\hat{b}_{k} \equiv Q_{k}^{T} b_{k}$, and all shrunk equations $\hat{A}_{k} x=\hat{b}_{k}$ are reassembled into the form (40). The number of equations in the shrunk form is thus reduced to $n_{1}+\cdots+n_{K}$.

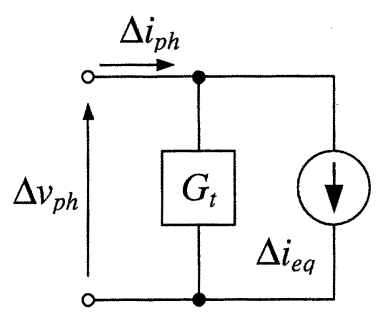
incremental values (a) equivalent circuit for

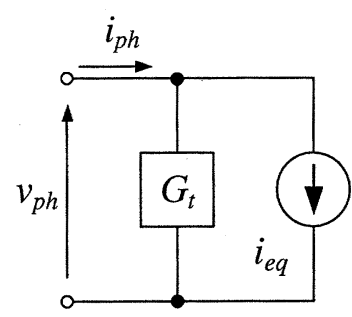

(b) equivalent circuit for full values
Fig. 3. Norton-type equivalent circuits used in an electromagnetic transient program.

\section{Time-Domain SimUlation}

\section{A. Time-Domain State Equations and Equivalent Circuit}

The $T$-periodic coefficients $a_{0}(t), \ldots, a_{N-1}(t)$ in (10) and $b_{0}(t), \ldots, b_{N-1}(t)$ in (11) are calculated by their Fourier coefficient matrices $A_{0}, \ldots, A_{N-1}$ and $B_{0}, \ldots, B_{N-1}$ in (19), which have been identified in the preceding section. Thus, we now have a reduced-order CF model of the external zone (8). Because most of electromagnetic transient programs use the trapezoidal rule of integration for the discretization of dynamic elements with respect to time, this paper uses the trapezoidal rule for the discretization of the CF model equivalent (8):

$$
\begin{gathered}
\Delta x(t)=E_{t}(t)\left\{F_{t}(t-\Delta t) \Delta x(t-\Delta t)+\frac{\Delta t}{2} B_{t}(t) \Delta u(t)\right. \\
\left.+\frac{\Delta t}{2} B_{t}(t-\Delta t) \Delta u(t-\Delta t)\right\}
\end{gathered}
$$

where

$$
E_{t}(t)=\left\{I-\frac{\Delta t}{2} A_{t}(t)\right\}^{-1}, \quad F_{t}(t)=I+\frac{\Delta t}{2} A_{t}(t) .
$$

It should however be noted that other discretization methods can also be used. Substituting (45) into the output equation (12) gives

$$
\Delta y(t)=G_{t}(t) \Delta u(t)+\Delta i_{e q}(t)
$$

where

$$
\begin{aligned}
G_{t}(t)= & \frac{\Delta t}{2} C E_{t}(t) B_{t}(t) \\
\Delta i_{e q}(t)= & C E_{t}(t) F_{t}(t-\Delta t) \Delta x(t-\Delta t) \\
& +\frac{\Delta t}{2} C E_{t}(t) B_{t}(t-\Delta t) \Delta u(t-\Delta t) .
\end{aligned}
$$

If the port voltage(s) $v_{p h}$ and current(s) $i_{p h}$ are taken as the input and output of the equivalent, (47) leads to the Norton-type equivalent circuit of Fig. 3(a) for incremental values. Expressing the incremental values $\Delta u$ and $\Delta y$ as the excursions from their underlying periodic steady state $\bar{u}$ and $\bar{y}$, and thus, $\Delta u=u-\bar{u}$ and $\Delta y=y-\bar{y}$, the following equation is obtained from (47):

$$
y(t)=G_{t}(t) u(t)+i_{e q}(t)
$$

where $i_{e q}(t)=\bar{y}-G_{t}(t) \bar{u}+\Delta i_{e q}(t)$. This is the Norton-type equivalent circuit of Fig. 3(b) for full values to be used in an electromagnetic transient program. Because the equivalent conductance $G_{t}(t)$ is $T$-periodic, the values of $G_{t}(t)$ over the period 


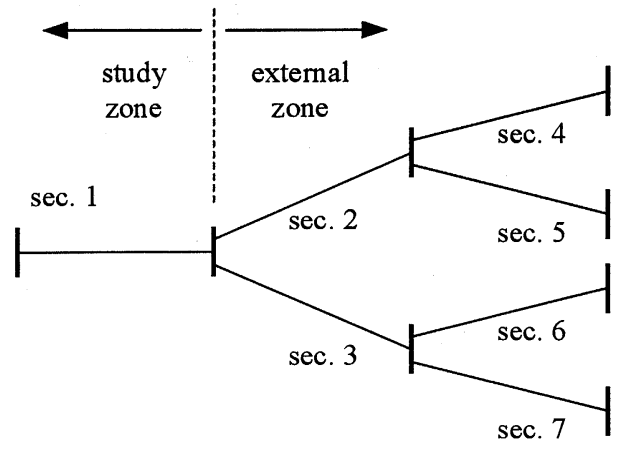

(a)

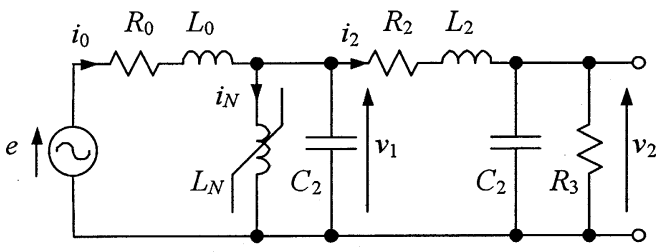

(b)

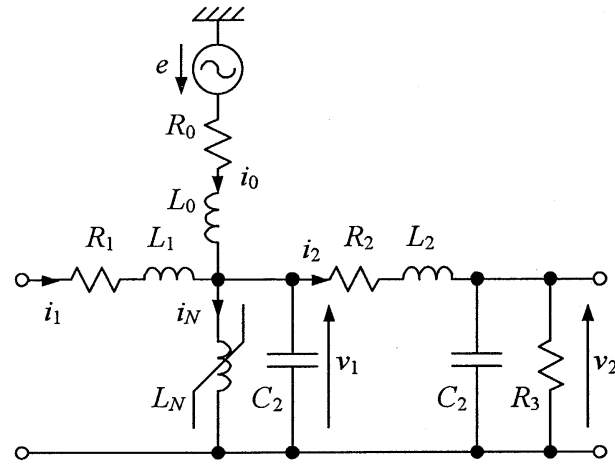

(c)

Fig. 4. Test Network. (a) Whole network. (b) First section. (c) Second to seventh sections.

TABLE I

PARAMETERS OF TEST NETWORK

\begin{tabular}{ccccccccc}
\hline $\begin{array}{c}\text { Sec. } \\
\#\end{array}$ & $\begin{array}{c}R_{0} \\
{[\Omega]}\end{array}$ & $\begin{array}{c}L_{0} \\
{[\mathrm{mH}]}\end{array}$ & $\begin{array}{c}R_{1} \\
{[\Omega]}\end{array}$ & $\begin{array}{c}L_{1} \\
{[\mathrm{mH}]}\end{array}$ & $\begin{array}{c}R_{2} \\
{[\Omega]}\end{array}$ & $\begin{array}{c}L_{2} \\
{[\mathrm{mH}]}\end{array}$ & $\begin{array}{c}C_{2} \\
{[\mu \mathrm{H}]}\end{array}$ & $\begin{array}{c}R_{3} \\
{[\Omega]}\end{array}$ \\
\hline 1 & 2.24 & 28.3 & - & - & 20.1 & 169 & 0.395 & 122 \\
2 & 2.97 & 28.0 & 1.99 & 59.0 & 21.6 & 127 & 0.827 & 104 \\
3 & 2.27 & 59.1 & 1.58 & 32.0 & 18.4 & 60.7 & 0.461 & 128 \\
4 & 1.47 & 44.9 & 4.05 & 53.8 & 24.4 & 79.1 & 0.514 & 101 \\
5 & 2.57 & 22.2 & 2.26 & 59.2 & 29.1 & 112 & 0.359 & 132 \\
6 & 1.01 & 25.7 & 2.69 & 40.8 & 29.0 & 129 & 0.580 & 120 \\
7 & 2.59 & 36.3 & 3.56 & 50.6 & 21.8 & 74.2 & 0.329 & 116 \\
\hline
\end{tabular}

may be calculated and stored prior to a transient simulation to gain computational speed.

\section{B. Balancing the Magnitudes of Coefficients}

In (46), the inverse of $\left\{I-(\Delta t / 2) A_{t}(t)\right\}$ must be evaluated. However, the magnitudes of identified denominator coefficients $a_{0}(t), \ldots, a_{N-1}(t)$ in $A_{t}$ can be significantly different and this may cause a rank deficiency in the inversion process. To avoid this, we balance the magnitudes of the coefficients. Let $a_{n, r m s}$
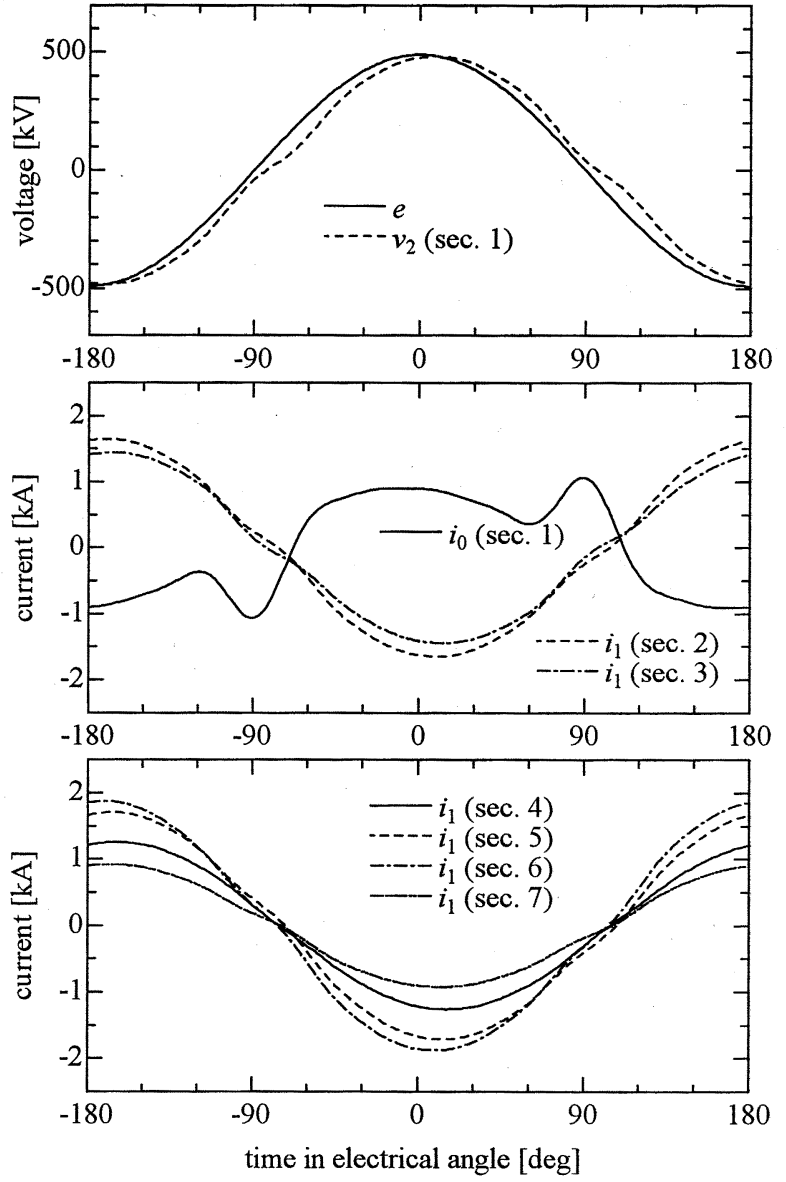

Fig. 5. Steady-state solution of Test Network.

be the rms value of $a_{n}(t)$ and $Q$ a diagonal transformation matrix defined by

$$
Q=a_{N-1, r m s} \operatorname{diag}\left(\frac{1}{a_{n, r m s}} ; n=0, \ldots, N-1\right) .
$$

The state vector $\Delta x$ is transformed by $\Delta z=Q \Delta x$ and the state equations (8) become

$$
\frac{d(\Delta z)}{d t}=A_{t}^{\prime} \Delta z+B_{t}^{\prime} \Delta u
$$

where $A_{t}^{\prime}=Q A_{t} Q^{-1}$ and $B_{t}^{\prime}=Q B_{t}$. The output equation (12) does not change. The magnitudes of the new $T$-periodic coefficients in $A_{t}^{\prime}$ are the same and this obviates the rank deficiency problem to evaluate (46).

\section{TEST CASE}

\section{A. Test Network}

Fig. 4 shows the test network used to demonstrate the identification performance of the proposed method. Fig. 4(a) shows a schematic diagram of the whole network consisting of seven sections. The first section, shown in detail in Fig. 4(b), is regarded as the study zone. Sections 2 to 7 are identical in topology, as shown in Fig. 4(c), and form the external zone. Each section has different circuit parameter values as shown in Table I. In the first section, the voltage source $e$ represents a $500-\mathrm{kV}$ bus voltage. $R_{0}$ and $L_{0}$ represent the combined leakage 


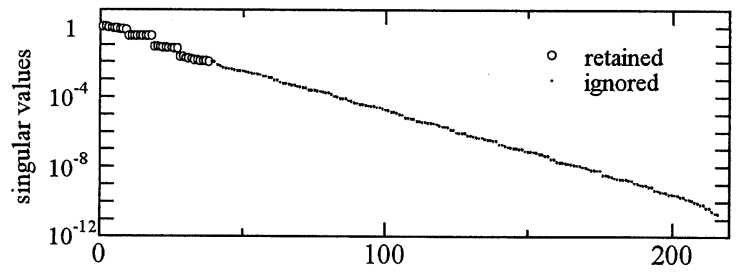

(a)

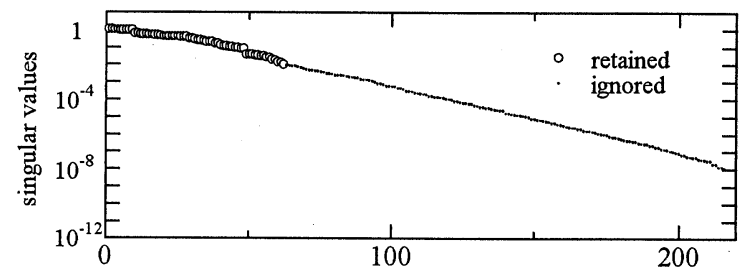

(b)

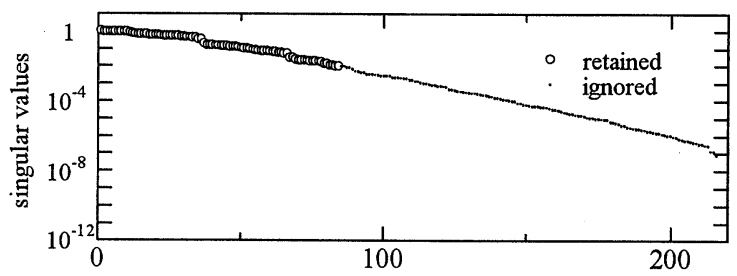

(c)

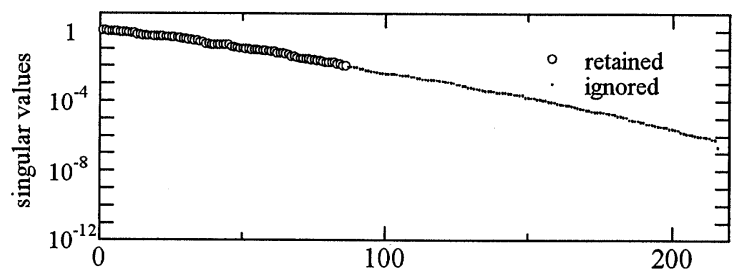

(d)

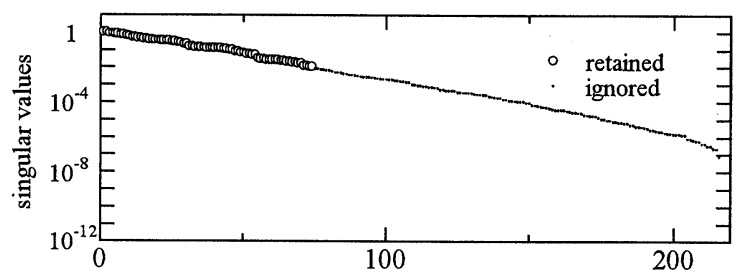

(e)

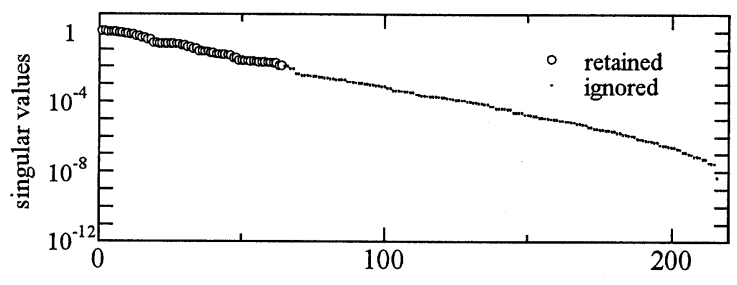

(f)

Fig. 6. Normalized singular values for each partition in the SVD-based vertical partitioning process preceding model order determination, where the horizontal axes show the singular value indices. The singular values are normalized with respect to the first one. The circles and dots show retained and ignored singular values, respectively; (a) partition \#1, (b) partition \#2, (c) partition \#3, (d) partition \#4, (e) partition \#5, (f) partition \#6.

impedance of the source $e$ and the corresponding step of a transformer. The transformer magnetizing branch $L_{N}$ is a non-

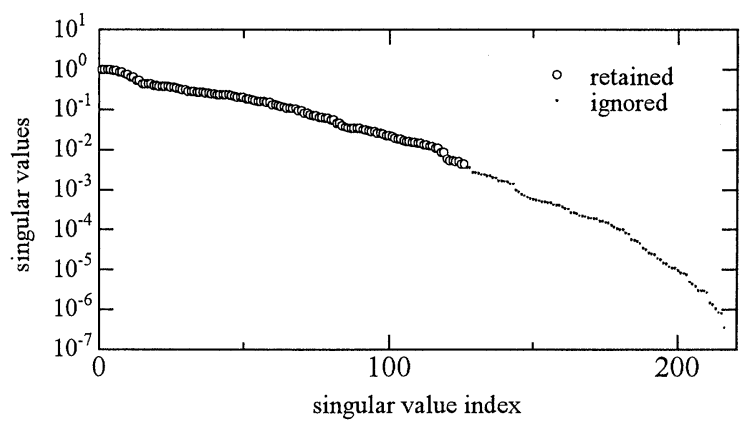

Fig. 7. Normalized singular values in the SVD-based order determination process at the initial iteration step. The singular values are normalized with respect to the first one. The circles and dots show retained and ignored singular values, respectively.

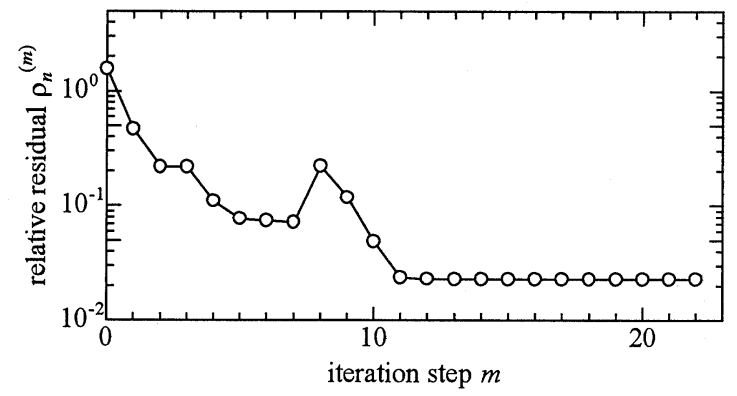

Fig. 8. Variation of relative residual with respect to iteration step.

linear inductor with a characteristic given by $i_{N}=0.01 \phi+$ $5 \times 10^{-69} \phi^{23}$ where $i_{N}$ and $\phi$ are the inductor current and flux, respectively. In the second to the seventh sections, the voltage sources identified by $e$ also represent $500-\mathrm{kV}$ bus voltages, and the source impedances are represented by $R_{0}$ and $L_{0}$. A transformer in each section is represented by $R_{1}, L_{1}$, and $L_{N}$, where $R_{1}$ and $L_{1}$ are the leakage impedance and $L_{N}$ is the magnetizing branch. The nonlinear characteristic of $L_{N}$ is given in the same way as in the first section. In each section, $R_{2}, L_{2}$, and $C_{2}$ represent a transmission line in the form of $\pi$ equivalent. Resistor $R_{3}$ represents the load in each section. The number of state variables in the external zone is 36 .

In this study, we assume a temporary overvoltage of 1.2 p.u. for all voltage sources $e$. Fig. 5 shows the steady-state solution of the test network obtained by the method proposed in [14] considering up to \pm 9 th harmonics. The nonlinear inductors create harmonics in the waveforms due to the overvoltage condition. It can be confirmed that the waveforms in the normal operation at 1 p.u., not shown here however, are almost sinusoidal. Based on the steady-state solution, the linearization of (3) and (4) is applied to the nonlinear state equations of the external zone, and the HDDTF matrix $H(s)$ is calculated in the form of frequency-scan data [4]. The frequency points are linearly sampled with 120 points between $0 \mathrm{~Hz}$ and $3 \mathrm{kHz}$.

\section{B. Identification and Frequency-Domain Results}

The HDDTF data are fitted with a reduced-order CF equivalent of Section III by the identification method described in Section IV. In the identification process, up to \pm 9 th harmonics are considered in the Fourier coefficient matrices $A_{n}$ and 

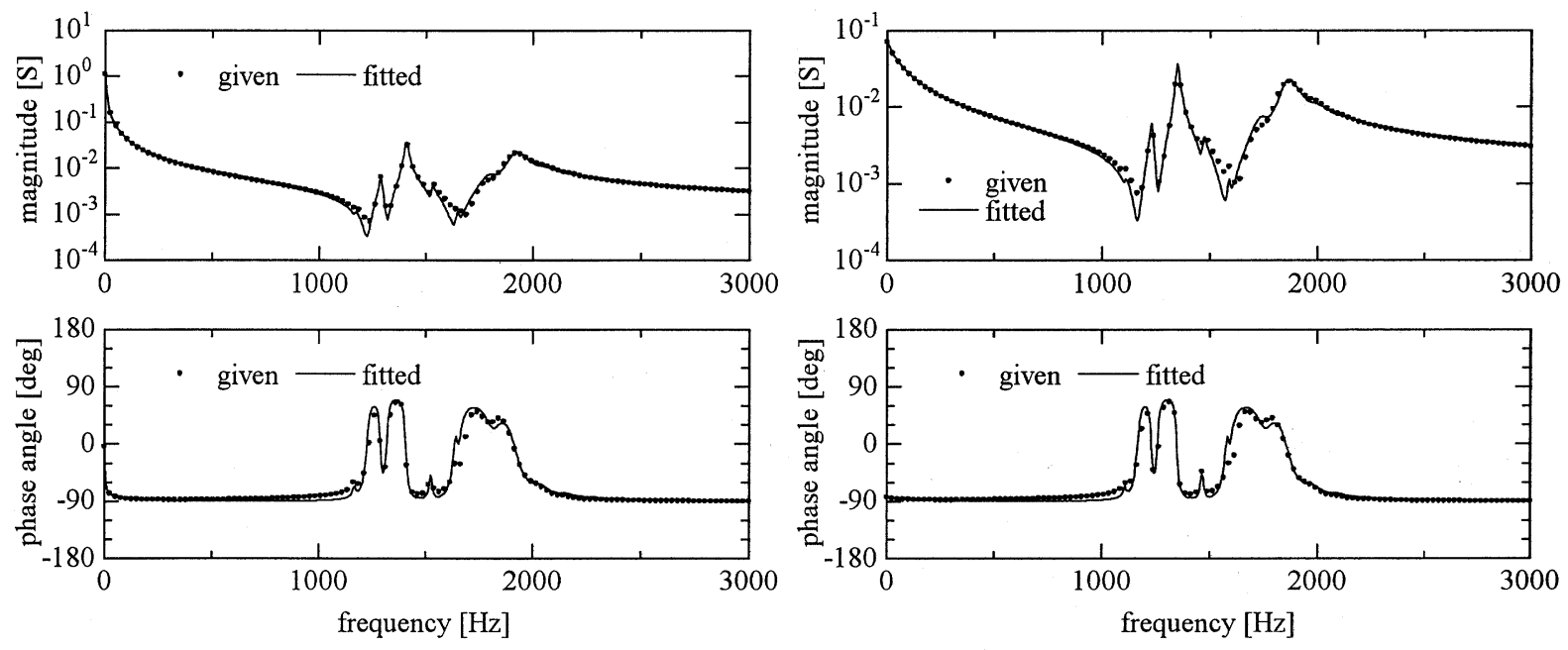

(a)

(b)
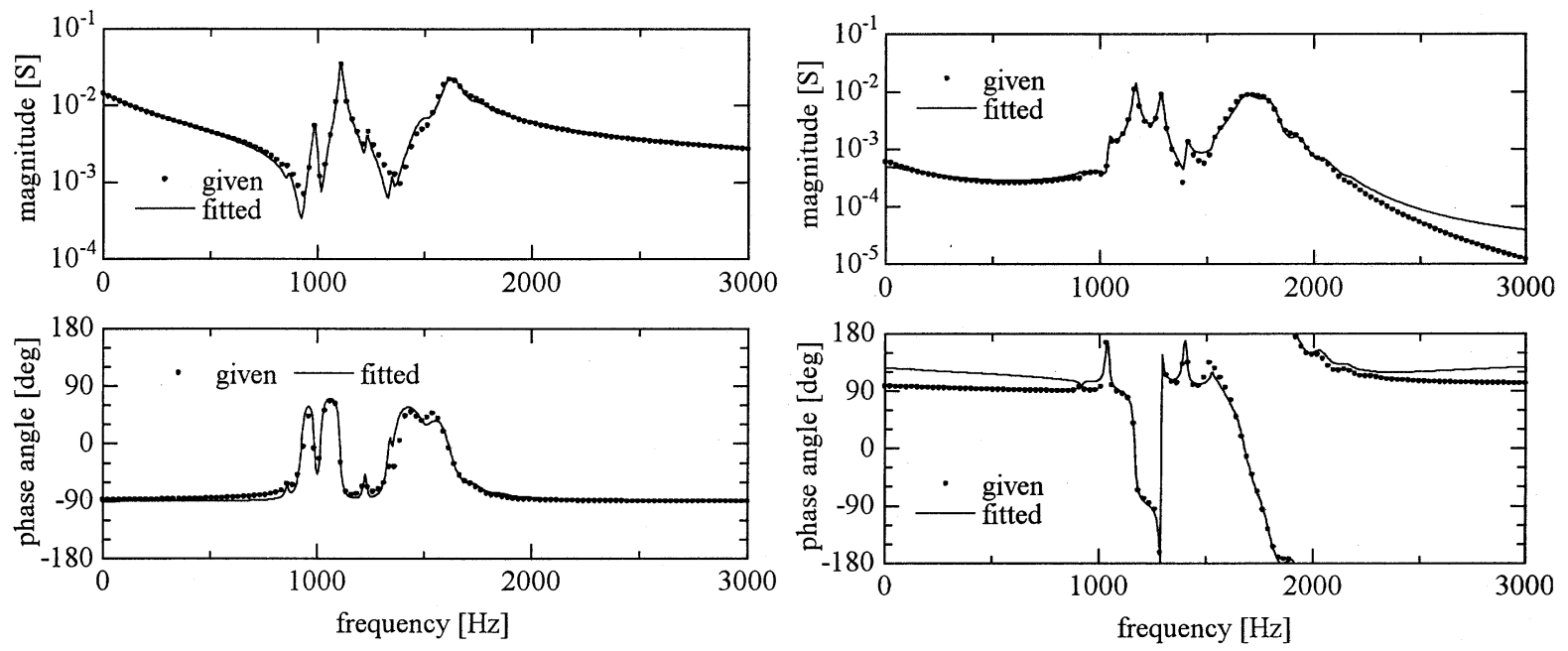

(c)

(d)

Fig. 9. Comparison of frequency scans of HDDTF matrices between "given" external zone and "fitted" (identified) CF equivalent. Element ( $i, j$ ) of HDDTF matrix $H(s)$ relates the $j$-th harmonic input to the $i$-th harmonic output; (a) $(0,0)$ element, (b) $(1,1)$ element, (c) $(5,5)$ element, (d) $(2,4)$ element.

$B_{n}$ in (19) $\left(N_{k}=10\right)$, and uniform weighting is applied to all frequency points in (25) [all $w_{p}$ 's are set to unity, and thus, (24) is used]. The user-defined relative error $\varepsilon$ for the SVD-based order determination process is set to $0.01(1 \%)$, and the initial model order is assumed to be 12 as a maximum acceptable order. Vertical partitioning is used to reduce the number of equations in (26), and the equations are uniformly divided into six partitions. The QR-based iterative solution process is terminated, when the relative difference of $\rho$ becomes less than $1 \times 10^{-6}$.

Fig. 6 shows retained and ignored singular values in the SVDbased partitioning process at the initial iteration step. This partitioning is performed to reduce the number of equations for the subsequent order determination process using SVD. Singular values larger than $\varepsilon=0.01$ shown by the circles are retained for the order determination process, while smaller singular values shown by the dots are ignored. Fig. 7 shows singular values in the SVD-based order determination process. Retained singular values which are larger than $\varepsilon=0.01$ are shown by the cir- cles, while ignored singular values by the dots. From the number of retained singular values, the model order is determined to be 7 . Because the test network exhibits half-wave symmetry, the number of retained singular values must be a multiple of $2\left(N_{k}-1\right)=18$, and thus, the total number of retained singular values is adjusted to 126 .

The QR-based iterative solution process follows the order determination. Fig. 8 shows the variation of the relative residual $\rho_{n}$ during the iteration process, where $\rho_{n}$ is given by normalizing $\rho$ with the Frobenius norm of the given HDDTF:

$$
\rho_{n}^{(m)}=\sqrt{\frac{\left\{\rho^{(m)}\right\}^{2}}{\sum_{p}\left\|H_{p}\right\|_{F}^{2}}} .
$$

The convergence of the iteration process is considered to be achieved at the 22 nd step, because $\left|\left(\rho^{(m)}-\rho^{(m-1)}\right) / \rho^{(m)}\right|$ becomes smaller than the given tolerance $1 \times 10^{-6}$. The final value of the relative residual $\rho_{n}$ is 0.0231 . We note that the value is 
close to the user defined relative error $\varepsilon$ given for the order determination process. Because the definitions of $\varepsilon$ and $\rho_{n}$ are different ( $\varepsilon$ is defined in terms of singular values while $\rho_{n}$ in terms of frequency-domain residual), their values do not turn out to be exactly the same. However, it is natural that if we truncate singular values based on $\varepsilon$, we obtain an equivalent with a residual $\rho_{n}$ of the same order as $\varepsilon$. This implies that a user can specify $\varepsilon$ roughly as the residual of a resultant equivalent.

Fig. 9 compares the frequency scans of the HDDTF matrices between the given external zone and the identified CF equivalent. Because the HDDTF matrices have 361 elements in this case (19 by 19), only some elements are shown here. Figs. 9(a)-(c) show the diagonal elements $(0,0),(1,1)$, and $(5$, $5)$, where element $(i, j)$ relates the $j$-th harmonic input to the $i$-th harmonic output. Fig. 9(d) shows the off-diagonal element $(2,4)$. At the high-frequency region, an error is observed in the element $(2,4)$, but it should be noted that the vertical axis is logarithmically scaled and the magnitude at that region is smaller in a least-squares sense. Considering the reduced order of the identified CF equivalent (from 36 to 7), the fitting observed in the frequency-scan comparison can be viewed as satisfactory.

\section{Time-Domain Simulation Results}

To demonstrate the time-domain accuracy of the identified equivalent, the following transient scenario is simulated: The test network is initially operating under a temporary overvoltage of 1.2 p.u. At $t=t_{1}$, the voltage source $e$ in the first section is suddenly reduced to 1.0 p.u., and then exactly one cycle later, at $t=t_{2}$, the same voltage source $e$ is increased again to 1.2 p.u. Both $t_{1}$ and $t_{2}$ correspond to $0^{\circ}$ in electrical angle as shown in Fig. 10.

In the simulation using the identified equivalent, only the study zone is represented in detail and the external zone is replaced by the equivalent. The state equations of the internal zone are formulated and then the equivalent in the form of (50) is added to the state equations. Because the state equations are nonlinear due to $L_{N}$ in the first section, the trapezoidal rule of integration combined with Newton-Raphson iteration is used, with a time step of $65.1 \mu \mathrm{s}$ ( 256 points per cycle). The simulation results, the waveforms of $e, v_{1}$, and $i_{2}$ in the first section, are shown in Fig. 10. The same transient scenario is also simulated when the full representation of the external zone is considered and the same integration method and time step are used. The results are superimposed in Fig. 10, where good agreement is illustrated between the results based on the full and the reduced-order representations of the external zone. Thus, the seventh order equivalent with periodic coefficients is able to reproduce the transient of the 36th order external zone taking into account the nonlinear effects at the steady-state operating point. It should also be noted that the equivalent is able to reproduce the correct nonsinusoidal steady state due to the periodic coefficients. Because of this, the subsequent transient is calculated accurately with the correct history values in (49).

\section{Efficiency}

The transient simulation with the equivalent for 20 cycles requires $38.3 \mathrm{~s}$ on a $600-\mathrm{MHz}$ Pentium III processor, while the
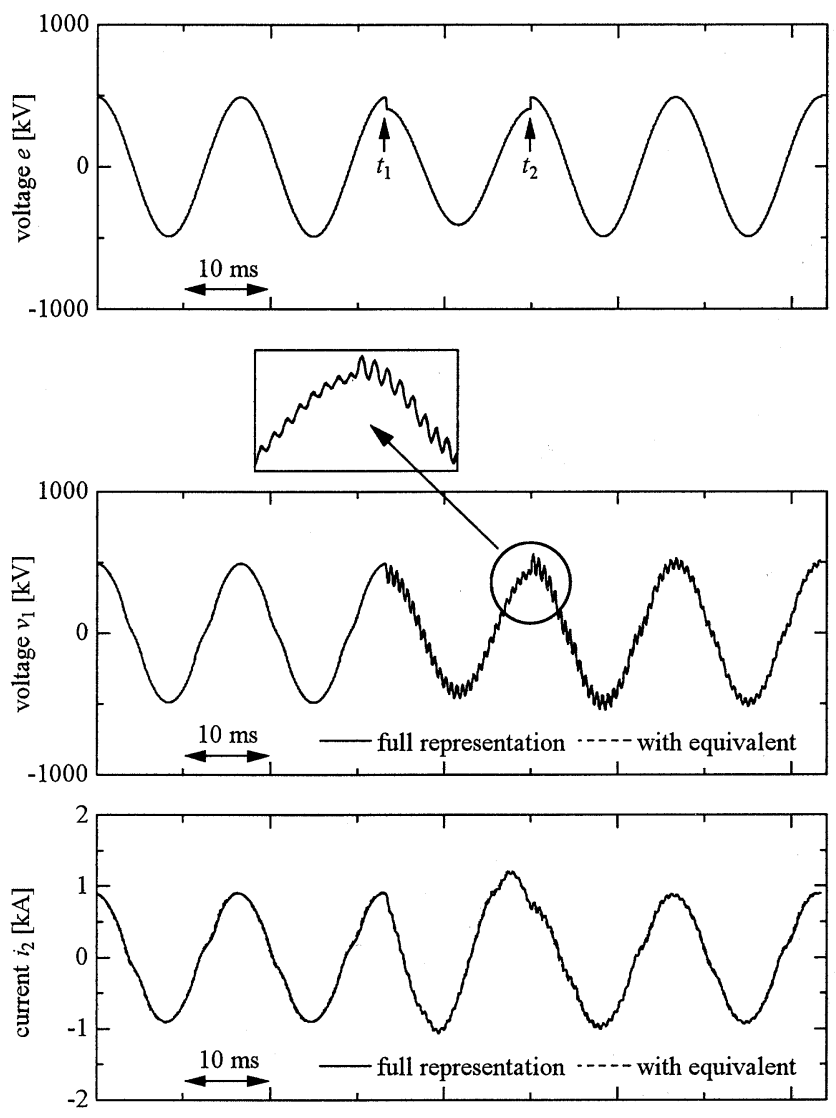

Fig. 10. Comparison of transient waveforms obtained using the identified equivalent and those obtained by the full representaion of the external zone. The waveforms of $e, v_{1}$, and $i_{2}$ in the first section are shown.

full representation requires $775 \mathrm{~s}$. The 20 -times faster computation is achieved by the equivalent due both to the reduced order and to the linearity of the equivalent. In the simulation with the equivalent, the values of $G_{t}(t)$ over a period are calculated and stored in memory prior to the transient simulation to gain computational speed as mentioned in Section V-A.

\section{CONCLUSIONS}

In this paper, a methodology for the identification of a reduced-order dynamic equivalent of a nonlinear network is proposed for the simulation of electromagnetic transients. The concept of HDDTF is used to characterize the network's transient behavior in the frequency domain and the frequency-scan data of the HDDTF are fitted by reduced-order companion-form state equations with periodic coefficients via a least-squares procedure. The fitting procedure determines an appropriate order of the companion form equivalent by an SVD-based algorithm and solves the least-squares problem iteratively using the QR algorithm supplemented with sequential weighting, column scaling, and vertical partitioning to improve the accuracy and efficiency. A test network is used to show the results of frequency-domain fitting and of time-domain transient simulation. The proposed method identifies a seventh order equivalent of the 36th order original system. The transient simulation results with the equivalent agree well with those by the full representation of the 


$A=\left[\begin{array}{c|c|c|c|c|c}C_{A_{N-1}, 1} & C_{B_{N-1}, 1} & \ldots & \ldots & C_{A_{0}, 1} & C_{B_{0}, 1} \\ \hline C_{A_{N-1}, 2} & C_{B_{N-1}, 2} & \ldots & \ldots & C_{A_{0}, 2} & C_{B_{0}, 2} \\ \hline \vdots & \vdots & & & \vdots & \vdots \\ \hline C_{A_{N-1}, p} & C_{B_{N-1}, p} & \ldots & \ldots & C_{A_{0}, p} & C_{B_{0}, p} \\ \hline \vdots & \vdots & & & \vdots & \vdots \\ \hline C_{A_{N-1}, P} & C_{B_{N-1}, P} & \ldots & \ldots & C_{A_{0}, P} & C_{B_{0}, P}\end{array}\right]$.

original system and a 20 times faster computational speed is achieved.

\section{APPENDIX}

\section{A. Building (26)}

First, we focus on the first term of the left-hand side of (24) (or, equivalently (25)). The matrix-matrix multiplication $W_{p}^{(m)} S_{p}^{n} A_{n}^{(m)} H_{p}$ is rearranged into the matrix-vector form $C_{A_{n}, p}^{\prime} x_{A_{n}}$ by placing each element of $W_{p}^{(m)} S_{p}^{n} A_{n}^{(m)} H_{p}$ into a row of $C_{A_{n}, p}^{\prime} x_{A_{n}}$. Since the coefficient matrix $C_{A_{n}, p}^{\prime}$ is complex-valued, it is rearranged into the form $C_{A_{n}, p} x_{A_{n}}$ to obtain the real-valued coefficient matrix defined by

$$
C_{A_{n, p}}=\left[\begin{array}{l}
\operatorname{Re}\left\{C_{A_{n}, p}^{\prime}\right\} \\
\operatorname{Im}\left\{C_{A_{n}, p}^{\prime}\right\}
\end{array}\right] .
$$

In the same manner, the second term $-W_{p}^{(m)} S_{p}^{n} B_{n}^{(m)}$ also results in the real-valued form $C_{B_{n}, p} x_{B_{n}}$. We then arrange the coefficient matrices $C_{A_{n}, p}$ and $C_{B_{n}, p}$ in the form

$$
A x \cong b
$$

where (See the equation at the top of the page). Here, each element of the right-hand side term $-W_{p}^{(m)} S_{p}^{N} H_{p}$ of (24) is put into a corresponding row of vector $b$. The above mentioned transformations can be performed in a systematic manner by the use of an incidence matrix to take into account the Toeplitz and Hermitian structures of the unknown matrices. The incidence matrix is also utilized to put the real and imaginary parts of the unknowns, instead of complex conjugate pairs, in vector $x$.

\section{REFERENCES}

[1] R. C. Dugan, M. F. McGranaghan, and H. W. Beaty, Electrical Power System Quality. New York: McGraw-Hill, 1996.

[2] J. Arrillaga, N. R. Watson, and S. Chen, Power System Quality Assessment. New York: Wiley, 2000.

[3] M. H. J. Bollen, Understanding Power Quality Problems: Voltage Sags and Interruptions, Piscataway, NJ, 2000, IEEE Press Power Eng. Series.

[4] T. Noda, A. Semlyen, and R. Iravani, "Harmonic domain dynamic transfer function of a nonlinear time-periodic network," IEEE Trans. Power Delivery, vol. 18, pp. 1433-1441, Oct. 2003.

[5] R. A. Rohrer, Circuit Theory: An Introduction to the State Variable Approach. New York: McGraw-Hill, 1971.

[6] B. C. Smith, J. Arrillaga, A. R. Wood, and N. R. Watson, "A review of iterative harmonic analysis for AC-DC power systems," IEEE Trans. Power Delivery, vol. 13, pp. 180-185, Jan. 1998.
[7] M. Farkas, Periodic Motions. New York: Springer-Verlag, 1994, vol. 104, Applied Mathematical Sciences.

[8] A. D’Angelo, Linear Time-Varying Systems: Analysis and Synthesis. Boston, MA: Allyn and Bacon, 1970.

[9] A. Semlyen, " $s$-domain methodology for assessing the small signal stability of complex systems in nonsinusoidal steady state," IEEE Trans. Power Syst., vol. 14, pp. 132-137, Feb. 1999.

[10] C. L. Lawson and R. J. Hanson, Solving Least Squares Problems. Philadelphia, PA: SIAM, 1995, vol. 15, Classics in Applied Mathematics.

[11] A. Björck, Numerical Methods for Least Squares Problems. Philadelphia, PA: SIAM, 1996.

[12] A. O. Soysal and A. Semlyen, "Practical transfer function estimation and its application to wide frequency range representation of trans-formers," IEEE Trans. Power Delivery, vol. 8, pp. 1627-1637, July 1993.

[13] A. Van der Sluis, "Condition numbers and equilibration of matrices," Numer. Math., vol. 14, pp. 14-23, 1969.

[14] A. Semlyen, E. Acha, and J. Arrillaga, "Newton-type algorithms for the harmonic phasor analysis of nonlinear power circuits in periodical steady state with special reference to magnetic nonlinearities," IEEE Trans. Power Delivery, vol. 3, pp. 1090-1098, July 1988.

Taku Noda (M'97) was born in Osaka, Japan, on July 4, 1969. He received the B.Sc., M.Sc., and Ph.D. degrees in engineering from Doshisha University, Kyoto, Japan, in 1992, 1994, and 1997, respectively.

Currently, he is a Visiting Scientist at the University of Toronto, ON, Canada. He was with DEI Simulation Software, Neskowin, OR, in 1994, and a Consultant with Bonneville Power Administration, Portland, OR, in 1995. In 1997, he joined Central Research Institute of Electric Power Industry (CRIEPI), where he is Research Scientist. His main research interest is the transient analysis of power systems.

Dr. Noda is a member of IEE of Japan.

Adam Semlyen (LF'97) was born in 1923 in Rumania. He received the Dipl.Ing degree from the Polytechnic Institute of Timisoara, Rumania, in 1950, and the Ph.D. degree from the Polytechnic Institute of Iasi, Rumania, in 1965.

He began his career in Timisoara with an electric power utility and held academic positions at the Polytechnic Institute. In 1969, he joined the University of Toronto, ON, Canada, where he is a Professor in the Department of Electrical and Computer Engineering, emeritus since 1968. His research interests include steady state and dynamic analysis as well as computation of electromagnetic transients in power systems.

Reza Iravani (SM'00) received the B.Sc. degree in electrical engineering in 1976 from Tehran Polytechnique University, Tehran, Iran. He received the M.Sc. and Ph.D. degrees in electrical engineering from the University of Manitoba, Winnipeg, Canada, in 1981 and 1985, respectively.

Currently, he is a Professor at the University of Toronto, ON, Canada. He began his career as a Consulting Engineer. His research interests include power electronics and power system dynamics and control. 